hep-th/0203228

\title{
A new class of Matrix Models arising from the $W_{1+\infty}$ Algebra
}

\author{
Henry David HERCE \\ Department of Physics, North Carolina State University, Raleigh NC 27695, \\ U.S.A. \\ Guillermo Raúl ZEMBA 円 \\ Physics Department, Comisión Nacional de Energía Atómica, Av.Libertador 8250, \\ (1429) Buenos Aires, Argentina and Universidad Favaloro, Solís 453, (1078) \\ Buenos Aires, Argentina
}

\begin{abstract}
We present a new class of hermitian one-matrix models originated in the $W_{1+\infty}$ algebra: more precisely, the polynomials defining the $W_{1+\infty}$ generators in their fermionic bilinear form are shown to expand the orthogonal basis of a class of random hermitian matrix models. The corresponding potentials are given, and the thermodynamic limit interpreted in terms of a simple plasma picture. The new matrix models can be successfully applied to the full bosonization of interesting one-dimensional systems, including all the perturbative orders in the inverse size of the system. As a simple application, we present the all-order bosonization of the free fermionic field on the one-dimensional lattice.
\end{abstract}

March 2002

\footnotetext{
${ }^{1}$ Fellow of CONICET, Argentina.
} 
The study of one-dimensional fermionic systems has recently attracted much attention due to progress in manufacturing of mesoscopic devices. Although much progress has been made in the theoretical understanding of the dynamics of these strongly correlated systems, many interesting questions remain still unanswered. One theoretical tool that is successfully used is the bosonization of the fermionic degrees of freedom [1]: for a given fermionic system in the thermodynamic limit, one considers the low-lying particle-hole excitations around the Fermi points. Linearization of the dispersion relation around these leads to a relativistic conformal field theory [2] of these gapless effective degrees of freedom. More recently, an extended bosonization procedure incorporating the subsequent non-linear corrections

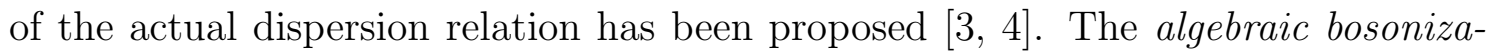
tion technique is an all-order bosonization approach based on the $W_{1+\infty}$ algebra, a characteristic fermionic symmetry [5] extending the conformal symmetry of the standard bosonization. The main advantage of this method is that it yields a systematic expansion of the full hamiltonian (including interactions) and all physical observables in powers of the momentum fluctuations around the Fermi points, involving algebraic procedures only.

In this letter, we present a one-parameter family of hermitian one-matrix models which originate in the $W_{1+\infty}$ algebra and are intimately connected to the algebraic bosonization of one-dimensional fermionic systems. Consider the Fock space of a $(1+1)$-dimensional relativistic Weyl fermionic field with creation and annihilation operators $a_{n}^{\dagger}$ and $a_{n}$, respectively ( $n$ is an integer that labels the momentum), satisfying $\left\{a_{n}^{\dagger}, a_{m}\right\}=\delta_{n, m}$. We define the $W_{1+\infty}$ operators $V_{n}^{k}(k=0,1,2, \ldots)$ in this specific basis [6, 7]:

$$
V_{n}^{k}=\sum_{r \in \mathbf{Z}} P_{n}^{k}(r): a_{r-n}^{\dagger} a_{r}:
$$

which satisfy the $W_{1+\infty}$ algebra [5],

$$
\left[V_{n}^{i}, V_{m}^{j}\right]=(j n-i m) V_{n+m}^{i+j-1}+q(i, j, n, m) V_{n+m}^{i+j-3}+\cdots+\delta^{i j} \delta_{n+m, 0} c d(i, n),
$$

where the structure constants $q(i, j, n, m)$ and $d(i, n)$ are polynomial in their arguments, $c$ is the central charge $(c=1$ for the single Weyl fermion), and the dots stand for a finite number of terms involving the operators $V_{n+m}^{i+j-1-2 k}$, with $k=0,1, \ldots,[(i+j-1) / 2]$, where the brackets denote the integer part function (the complete expression of (22) is given in [6]). The meaning of the operators $V_{n}^{k}$ is as follows: they parametrize particle-hole excitations near the Fermi points, such that the index $n$ accounts for the fixed momentum transfer, whereas the index $k$ denotes the geometric moments (i.e., "multipole moments") of the deformation of

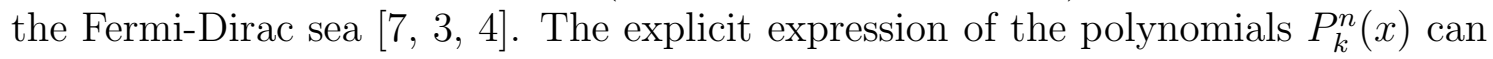
be inferred directly from the $W_{1+\infty}$ algebra [8]: 


$$
P_{n}^{k}(x)=\frac{(-1)^{k}}{\left(\begin{array}{c}
2 k \\
k
\end{array}\right)} \sum_{j=0}^{n}(-1)^{j}\left(\begin{array}{c}
n \\
j
\end{array}\right)^{2}(n+1-x)_{k-j}(x)_{j}
$$

where $(a)_{n}=\prod_{\ell=0}^{n-1}(a+\ell)$ denotes the Pochhammer symbol. Some properties can be deduced from (3): i) the polynomials have a definite parity with respect to the reflection about $x=1 / 2$ : even $n$ (resp. odd) correspond to even (odd) polynomials. ii) for $n$ large enough, the zeroes of $P_{n}^{k}(x)$ are located on the line $x=1 / 2+i \zeta$, with $\zeta$ real, and for pure imaginary values of $n$, all zeroes fall along this line. For $k$ even, all zeroes come in complex conjugate pairs, and for $k$ odd the same is true except for a single zero at $x=1 / 2$. However, the most important consequence of (3) is that the $P_{n}^{k}(x)$ satisfy a three-term recurrence relation:

$$
x P_{n}^{k}(x)=P_{n}^{k+1}(x)+\frac{1}{2} P_{n}^{k}(x)+\frac{k^{2}\left(n^{2}-k^{2}\right)}{4\left(4 k^{2}-1\right)} P_{n}^{k-1}(x) .
$$

This property has the important consequence of leading to a new class of random matrix models associated to the $W_{1+\infty}$ algebra, as we shall show in the following.

Matrix models constitute another important tool when studying systems displaying universal behavior in the thermodynamic limit [9]. There are many know examples in which the symmetries that characterize the relevant effective degrees of freedom of a given system are simply encoded in a specific random matrix model, which posses the advantage of being a simpler $(0+1)$-dimensional theory. Consider a generic random one-matrix model, defined by its partition function:

$$
Z(N)=\int d^{N^{2}} \Phi \exp [-\operatorname{Tr} V(\Phi)]
$$

where $\Phi$ is an $N \times N$ Hermitian matrix, Tr is the trace, ane the real function $V(\Phi)$ is the potential. Eventually, we shall be interested in the limit $N \rightarrow \infty$, which is the relevant thermodynamic limit. The matrix $\Phi$ has real eigenvalues $x_{i}, i=1, \ldots, N$, in terms of which the partition function can be written as [9]:

$$
Z(N)=\Omega(N) \int \ldots \int \prod_{i=1}^{N} d x_{i} \Delta_{N}^{2}(x) \exp \left(-\sum_{i=1}^{N} V\left(x_{i}\right)\right)
$$

where $\Delta_{N}(x)=\prod_{i<j}\left(x_{j}-x_{i}\right)$ and $\Omega(N)=(2 \pi)^{N(N-1) / 2} / \prod_{k=1}^{N} k$ ! is the volume of the "angular" (non-diagonal) entries of $\Phi$ [9]. A general strategy to compute $Z(N)$ is to factorize the $N$ integrals on the r.h.s. of (包), which can be done provided there exists a set of orthogonal polynomials $\mathcal{P}_{k}(x)$ satisfying:

$$
\int_{-\infty}^{\infty} d x \mathrm{e}^{-V(x)} \mathcal{P}_{k}(x) \mathcal{P}_{l}(x)=h_{k} \delta_{k, l}
$$


In turn, this condition can be met provided the polynomials $\mathcal{P}_{k}(x)$ satisfy a threeterm recurrence relation given by:

$$
x \mathcal{P}_{k}(x)=\mathcal{P}_{k+1}(x)+S_{k} \mathcal{P}_{k}(x)+R_{k} \mathcal{P}_{k-1}(x),
$$

where $S_{k}$ and $R_{k}$ are specific to the matrix model [9]. A crucial equality that relates the potential with the recursion relation is $R_{k}=h_{k} / h_{k-1}$, and the partition function is given by:

$$
Z(N)=\Omega(N) N ! \prod_{k=0}^{N-1} h_{k}=\Omega(N) N ! h_{0}^{N} \prod_{k=0}^{N-1} R_{k}^{N-k}
$$

In the following, we shall focus in the set of polynomials (3), and show that they are orthogonal with respect to an specific measure which is then used to define the associated matrix model. One anticipates, therefore, an infinite family of models parametrized by the momentum $n$, and defined by the family of potentials $V_{n}(x)$. Given the location of the zeroes of the polynomials (3), it is natural to perform a "Wick rotation" in $x$ and define $\mathcal{P}_{n}^{k}(x)=i^{k} P_{n}^{k}(-i x+(n+1) / 2)$ as the set of $W_{1+\infty}$ orthogonal polynomials. They posses a definite parity under reflection in $x$; the first few are: $\mathcal{P}_{n}^{0}(x)=1, \mathcal{P}_{n}^{1}(x)=x, \mathcal{P}_{n}^{2}(x)=x^{2}+\left(n^{2}-1\right) / 12, \mathcal{P}_{n}^{3}(x)=x^{3}+x\left(3 n^{2}-7\right) / 20$. The recursion relation (田) now takes the form:

$$
x \mathcal{P}_{n}^{k}(x)=\mathcal{P}_{n}^{k+1}(x)+\frac{k^{2}\left(k^{2}-n^{2}\right)}{4\left(4 k^{2}-1\right)} \mathcal{P}_{n}^{k-1}(x),
$$

which implies the existence of the set of potentials $V_{n}(x)$ making them orthogonal. These functions are readily found once one recognizes that the polynomials $\mathcal{P}_{n}^{k}(x)$ are Hahn polynomials, for which the orthogonality relations are known[10]. There are, in fact, two classes of Hahn polynomials: discrete and continuous. The first case arises for $n$ integer, such that there is a finite set of polynomials, since the recursion relation (10) stops at $k=n$. However, given (11), we are interested in the case for which the family is infinite: this is the continuous case, that arises for $n$ not an integer. For our purposes, it is convenient to consider the values $n=i p$, $p=0,1,2, \ldots$ (i.e., imaginary momentum) since this yields a positive and nondegenerate inner product. This condition can be viewed as the Wick rotation in momentum space that corresponds to the one previously performed in $x$. Under these conditions, the orthogonality relations are given by [10]:

$$
\begin{gathered}
\int_{-\infty}^{\infty} d x \quad g_{p}(x) \quad \mathcal{P}_{i p}^{k}(x) \mathcal{P}_{i p}^{l}(x)=\frac{|\Gamma(k+1+i p)|^{2}}{(2 k+1)\left(\begin{array}{c}
2 k \\
k
\end{array}\right)^{2}} \delta_{k, l} \\
g_{p}(x)=\frac{\pi}{\cosh (2 \pi x)+\cosh (p \pi)} .
\end{gathered}
$$




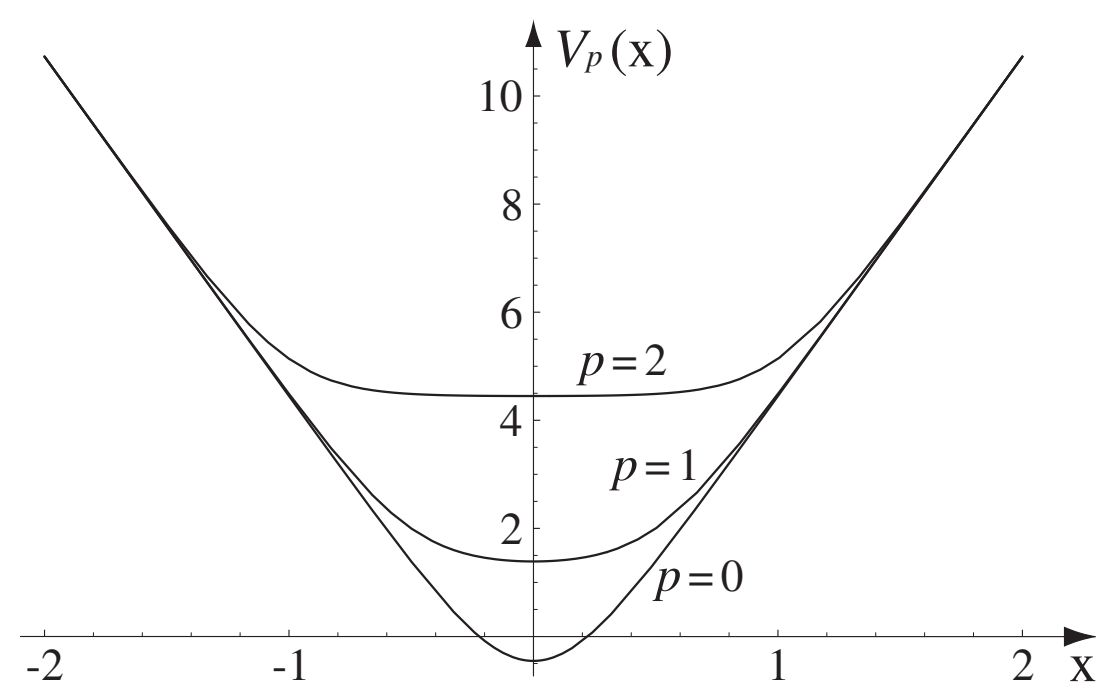

Figure 1: The potentials $V_{p}(x)$ as a function of $x$ for $p=0,1,2$. Note the asymptotic linear behavior discussed in the text.

From this expression, one extracts the family of potentials $V_{p}(x)$ defining the matrix models as in (5):

$$
V_{p}(x)=-\ln g_{p}(x)=\ln \left[\frac{\cosh (2 \pi x)+\cosh (p \pi)}{\pi}\right] \quad, \quad p=0,1,2, \ldots,
$$

which asymptotically behave as $V_{p}(x) \simeq 2 \pi|x|-2 \ln (2 \pi)$ for $|x|>\gamma_{p} / \pi, \gamma_{p}=$ $\ln [2 \pi \cosh (p \pi)]$ (see figure 1). From (11), one also knows that the norm of the $k-t h$ polynomial in the $p-t h$ matrix model is:

$$
h_{p}^{k}=\frac{|\Gamma(k+1+i p)|^{2}}{(2 k+1)\left(\begin{array}{c}
2 k \\
k
\end{array}\right)^{2}}
$$

which is in agreement with the recursion relation (10) through the equality $R_{p}^{k}=$ $h_{p}^{k} / h_{p}^{k-1}=k^{2}\left(k^{2}+p^{2}\right) / 4\left(4 k^{2}-1\right)$. From (13), one can therefore define the partition function $Z_{p}(N)$ and free energy density $f_{p}(N)$ for the $p-t h$ model as:

$$
Z_{p}(N)=\Omega(N) N ! \prod_{k=0}^{N-1} h_{p}^{k}=\exp \left(N^{2} f_{p}(N)\right)
$$

The asymptotic form of $f_{p}(N)$ in the thermodynamic limit $N \rightarrow \infty$ is:

$$
f_{p}(N)=\frac{1}{2} \ln N-\left(\frac{3}{4}+\frac{1}{2} \ln \frac{8}{\pi}\right)+O(\ln N / N)
$$

independent of $p$ to the given order. 
The thermodynamic limit leads also to a simple physical picture in terms of a fictitious one-dimensional plasma: consider the partition function (5) for the potential (12) (we consider for simplicity the case $p=0$ ). The asymptotic form of $V_{0}(x)$ is linear, and suggests the definition of a new scaling variable $y=x / N$, appropriate in the limit $N \rightarrow \infty$. This implies that $V_{0}(x) \simeq 2 \pi N|y|-2 \ln (2 \pi)$ for $|y|>1 / \Lambda$, with $\Lambda=\pi N / \gamma_{0}$. In the scaling limit, $y$ is defined in the entire real axis, except for a small region of the size of the cutoff $1 / \Lambda$ around the origin. We can write the partition function approximately as:

$Z_{0}(N) \simeq \frac{\Omega(N)}{N^{N}} \int \prod_{i=1}^{N} d y_{i} \exp \left[-N\left(2 \pi \sum_{i=1}^{N}\left|y_{i}\right|-\frac{1}{N} \sum_{i<j} \ln \left(y_{i}-y_{j}\right)^{2}+f(N)\right)\right]$,

where $f(N)=(N-1) \ln N+2 \ln (2 \pi)$. In the large $N$ limit, $Z_{0}(N)$ is dominated by the saddle-point configurations $\left\{y_{1}, \ldots, y_{N}\right\}$ satisfying the equations:

$$
2 \pi \operatorname{sg}\left(y_{i}\right)=\frac{2}{N} \sum_{j, j \neq i} \frac{1}{y_{i}-y_{j}}, \quad i=1, \ldots, N,
$$

where $\operatorname{sg}(y)$ is the sign function. The scaled variable $y$ is appropriate for taking the continuum limit: $y_{i} \rightarrow y, \sum_{i}(\ldots) / N \rightarrow \int d y \rho(y)(\ldots)$, where $\rho(y)$ is the density function. The saddle-point equations are:

$$
\begin{aligned}
\pi \operatorname{sg}(y) & =\mathcal{P} \int_{-\infty}^{+\infty} \frac{\rho\left(y^{\prime}\right)}{y-y^{\prime}} d y^{\prime}, \\
\int_{-\infty}^{\infty} \rho\left(y^{\prime}\right) d y^{\prime} & =1
\end{aligned}
$$

where $\mathcal{P}$ denotes the Cauchy principal part of the integral, and the second equality is the normalization condition. These equations give a semiclassical physical interpretation to our system: they describe a system of fictitious equally charged "particles", located on a one-dimensional rod, and subject to a neutralizing background. The particles repel each other with a two-dimensional Coulomb force, while they are attracted with constant force to an infinite two-dimensional uniformly charged plane, that is perpendicular to the rod. One can find a solution to equations (19) by assuming that the system is symmetric under $y \rightarrow-y$, and therefore the total number of "particles" is even, with $N / 2$ of them on each side of the infinite charged plane. Therefore, one seeks for a solution of the form $\rho(y)=\rho_{+}(y) E(y)+\rho_{-}(y) E(-y)$, with $E(y)$ the Heaviside function. In the following we shall focus on one side only, e.g. $(+)$. As it is customary, one introduces the function

$$
F_{+}(y)=\int_{-\infty}^{\infty} \frac{\rho\left(y^{\prime}\right)}{y-y^{\prime}} d y^{\prime}
$$

defined on the complex half-plane $\operatorname{Re}(y)>0$. Assuming that this function has one cut running along the real axis from 0 to $\alpha$, one can solve (19) by a standard 
procedure [9], yielding:

$$
F_{+}(y)=\pi-\pi \sqrt{\frac{y-\alpha}{y}},
$$

From (21) one determines the density:

$$
\rho_{+}(y)=\sqrt{\frac{\alpha-y}{y}},
$$

where $\alpha=1 / \pi$ is determined from the normalization condition (19). One can verify that along the cut and close to its extreme $y \simeq \alpha$, the behavior of $\rho_{+}(y)$ is in agreement with the expected one for a linear background potential [9].

Let us now focus on the application of the previous results to one-dimensional condensed matter models. Consider a free non-relativistic fermionic field on a onedimensional lattice of unit spacing. We shall outline the effective field theory formulation of this problem in terms of the $W_{1+\infty}$ symmetry generators [i]]. While this description does not appear as necessary in the free theory, it becomes rather advantageous when including the effects of the interactions, as, for example, in the $X X Z$ Heisenberg model. We shall postpone the full discussion of the effects of the interactions for a forthcoming publication, but remark here that the scope of our approach is to develop a bosonization scheme valid to all orders in the expansion parameter, which is the inverse size of the system, given by $N$. In the thermodynamic limit, the non-relativistic field can be expanded in the basis of the relativistic Weyl field, and the Fermi sea interpreted as a Dirac sea 四. Moreover, the free hamiltonian $\mathcal{H}$ can be written as the sum of two independent terms, $\mathcal{H}_{+}$and $\mathcal{H}_{-}$, describing the fluctuations around the right $(+)$ and left $(-)$ Fermi points at momenta $\pm n_{F}$, respectively, with $n_{F}=(N-2) / 4$; i.e., we consider the ground state of the system to be at half-filling, for simplicity. Both terms are decoupled, up to global (zero-mode) constraints. Therefore, we shall consider $\mathcal{H}_{+}$only in the following:

$$
\mathcal{H}_{+}=-\sum_{r=-\infty}^{+\infty} f\left(n_{F}+r\right): a_{r}^{\dagger} a_{r}: \quad, \quad f(n)=\cos \left(\frac{2 \pi}{N} n\right)
$$

where $a_{r}$ are the fermionic Fock operators of the Weyl field, obtained from the original ones by shifting the momentum index, and $r$ is integer. The dispersion relation around the Fermi point given by $f\left(n_{F}+r\right)$ is non-linear, and can be expanded in terms of the zero-momentum $W_{1+\infty}$ polynomials (3) as [4:

$$
\mathcal{H}_{+}=\sum_{k=0}^{\infty} c_{k} V_{0}^{k}
$$

where the coefficients $c_{k}$ are such that:

$$
\sin \left[\frac{2 \pi}{N}\left(r-\frac{1}{2}\right)\right]=\sum_{k=0}^{\infty} c_{k} P_{0}^{k}(r) .
$$


Making the change of variables $r \rightarrow(-i r+1 / 2)$ and passing to the basis $\mathcal{P}_{0}^{k}(r)$ allows us to determine $c_{k}$ using the orthogonality relation (7):

$$
c_{k}=\frac{i^{k}}{h_{0}^{k}} \int_{-\infty}^{+\infty} d x g_{0}(x) \sin \left(\frac{2 \pi}{N} r\right) \mathcal{P}_{0}^{k}(x) \quad k=0,1,2, \ldots
$$

where for convenience we have made the redefinition $2 \pi / N \rightarrow i 2 \pi / N$, so as to deal with regular expressions; this analytic continuation should be undoed at the end of the computations. From (26) one immediately concludes that $c_{2 n}=0$ for $n$ integer, and defines $C_{k}=c_{2 k+1}(k=0,1,2, \ldots)$ for the other cases, which can be worked out by considering the set of integrals:

$$
I_{n}(y)=\int_{-\infty}^{+\infty} d x g_{0}(x) \mathrm{e}^{i x y} \mathcal{P}_{0}^{n}(x), n=0,1,2, \ldots
$$

As a consequence of (10), they satisfy the recursion relations:

$$
I_{n+1}(y)=-i \frac{d I_{n}(y)}{d y}-R_{0}^{n} I_{n-1}(y) \quad, \quad I_{0}(y)=\frac{y}{2 \sinh (y / 2)},
$$

which are formally solved by $I_{n}(y)=\mathcal{P}_{0}^{n}\left(-i \frac{d}{d y}\right) I_{0}(y)$. The coefficients (26) are then readily determined yielding:

$$
C_{k}=\frac{(-1)^{k}}{h_{0}^{2 k+1}} I_{2 k+1}\left(\frac{2 \pi}{N}\right), k=0,1,2, \ldots
$$

As and example, after analytically continuing back $\pi / N \rightarrow-i \pi / N$ as explained above,

$$
C_{1}=\frac{6}{\sin (\pi / N)}\left[1-\frac{\pi}{N \tan (\pi / N)}\right]=\frac{2 \pi}{N}+\frac{7}{120}\left(\frac{2 \pi}{N}\right)^{3}+O\left\{\left(\frac{2 \pi}{N}\right)^{5}\right\},
$$

which reproduces the results of [4]. We have also verified the leading term in $C_{2}=$ $-(2 \pi / N)^{3} / 6+\ldots$ yields the correct result.

Finally, we remark that the matrix models considered in this letter belong to the class of models with non-polynomial potentials, which include also the Penner [12 and Frustrated Spherical [13] models. According to the 't Hooft correspondence, one can associate to each matrix model a tiling of a two-dimensional random surface, such that the the number of sides and the ways of gluing the elementary poligons are determined by the algebraic powers of the monomials that define the potential [11] [9]. The class of matrix models with non-polynomial potentials possess the characteristic property of having an unbounded set of polygons and gluing vertices among them. We conclude by remarking that it would be interesting to explore whether other infinite-dimensional Lie algebras ecould give rise to further new classes of random matrix models.

GZ acknowledges the hospitality of the Abdus Salam Center for Theoretical Physics (Italy) and the Physics Department and I.N.F.N. of Florence (Italy). 


\section{References}

[1] See, for example: E. Fradkin, Field Theories of Condensed Matter Systems, Addison-Wesley (1991); H. J. Schulz, in "Proceedings of Les Houches Summer School LXI", ed. E. Akkermans, G. Montambaux, J. Pichard, et J. Zinn-Justin, Elsevier, Amsterdam (1995), p.533, cond-mat/9503150; J. Voit,Repts. on Prog. Phys. 58 (1995) 977, cond-mat/9510014.

[2] For a textbook see: P. Di Francesco, P. Mathieu and D. Senechal, Conformal Field Theories, Springer-Verlag, (1996).

[3] R. Caracciolo, A. Lerda and G. R. Zemba, Phys. Lett. B 352 (1995) 304; see also R. Caracciolo, M. Frau, A. Lerda and G. R. Zemba hep-th/9604009, M. Maule, S. Sciuto, Mod. Phys. Lett. A 12 (1997) 2153.

[4] M. Frau, A. Lerda, S. Sciuto and G. R. Zemba, Int. J. Mod. Phys. A 12 (1997) 4611; see also Int. J. Mod. Phys. A 12 (1997) 1113.

[5] For a review see: X. Shen, Int. J. Mod. Phys. 7 A (1992) 6953; P. Bouwknegt and K. Schoutens, Phys. Rept. 223 (1993) 183.

[6] V. Kac and A. Radul, Comm. Math. Phys. 157 (1993) 429; E. Frenkel, V. Kac, A. Radul and W. Wang, Comm. Math. Phys. 170 (1995) 337.

[7] A. Cappelli, C. A. Trugenberger and G. R. Zemba, Ann. Phys. 246 (1996) 86.

[8] H. Awata, M. Fukuma, Y. Matsuo and S. Odake, Prog. Theor. Phys. Suppl. 118 (1995) 343.

[9] D. Bessis, C. Itzykson, G. Parisi and J. B. Zuber, Adv. Appl. Math. 1 (1980) 109; C. Itzykson and J. B. Zuber, J. Math. Phys. 21 (1980) 411; For a review see, P. Di Francesco, P. Ginsparg and J. Zinn-Justin, Phys. Rep. 254 (1995) 1.

[10] R. Askey, J. Math. Phys. A: Math. Gen 18 (1985) L1017; H. T. Koelink, "On Jacobi and continuous Hahn polynomials", math.CA/9409230. For a review, see R. Koekoek and R. F. Swarttouw, R. F. The Askey-Scheme of Hypergeometric Orthogonal Polynomials and its q-Analogue, Technische Universiteit Delft, Faculty of Technical Mathematics and Informatics Report 98-17 (1998); ftp://www.twi.tudelft.nl/publications/tech-reports/1998/DUTTWI-98-17.ps.gz.

[11] G. 't Hooft, Nucl. Phys. B 72(1974) 461.

[12] J. Distler and C. Vafa, Mod. Phys. Lett. A 6 (1991) 259.

[13] A. Cappelli and F. Colomo, J. Phys. A 31 (1998) 3141. 